\title{
Numerical simulations of impulsively generated mass density perturbations in a solar coronal loop
}

\author{
M. Selwa and K. Murawski \\ Institute of Physics, UMCS, ul. Radziszewskiego 10, 20-031 Lublin, Poland \\ e-mail: mselwa@kft.umcs.lublin.pl \\ Received 24 March 2004 / Accepted 9 June 2004

\begin{abstract}
Non-symmetric oscillations in a solar coronal loop are numerically studied in the limit of a two-dimensional plasma. The obtained numerical results show that impulses, which are launched in the ambient plasma, excite oscillations in mass density profiles that reveal asymmetry in a cross-section of the loop. This asymmetry is a consequence of the fact that the loop oscillates as a whole. This feature has not been observed in the earlier numerical studies of impulsively generated waves, and it is important for the detecion of the spatial location of the source of the loop oscillations. It is also important for the observations because the density perturbations can produce corresponding perturbations of EUV or thermal X-ray emission.
\end{abstract}

Key words. Sun: corona - Sun: oscillations - magnetohydrodynamics (MHD)

\section{Introduction}

Oscillations in solar coronal loops have been observed for a few decades (see the reviews by Aschwanden 2003; Wang 2004). The importance of such oscillations lies in their potential for the diagnostics of coronal structure (magnetic field strength, gas density, etc.) through coronal seismology and their potential for heating the corona (Roberts et al. 1984; Roberts 2000; Nakariakov 2003; Nakariakov et al. 2004). However, before this potential can be realized a number of theoretical aspects of coronal loop oscillations need to be studied. The present investigation which is based on numerical modeling of MHD wave processes, aims to contribute to this topic.

One of the open questions regards the excitations of the various types of coronal waves that have now been observed. Here we consider the excitation of the transverse and vertical waves observed by TRACE (Aschwanden 2003; Wang \& Solanki 2004) within the context of the models developed a few years ago by Murawski \& Roberts (1993, 1994), Murawski et al. (1998), and recently by Nakariakov \& Roberts (1995), Cooper et al. (2003) and Nakariakov et al. (2004), i.e. we consider impulsively excited waves. Such an excitation may be due to a flare or any other process leading to a sudden release of energy. We employ an adaptation of a modern numerical code and use a fine spatial and temporal resolution. These improvements lead to qualitatively new results which correspond to impulsive excitation of characteristic mass density fluctuations. Note that the usual kink mode does not perturb the density in the same fashion (Nakariakov \& Roberts 1995). The density fluctuations that are associated with kink and sausage waves were seen recently by Cooper et al. (2003) and Wang \& Solanki (2004).
One of our aims is to contribute to the development of the theory-led techniques for the search of the waves in observational data. The new numerical results are important from the observational point of view as they reveal that the line intensity signal should be stronger on the side of a loop. The results are important in coronal heating and seismology of the solar corona.

The paper is organized as follows. A simple model of a coronal loop is developed in Sect. 2. The next section contains results of the numerical simulations for impulsively generated MHD waves in a coronal loop. This paper is concluded by the presentation and discussion of the main results in Sect. 4.

\section{Physical model of a coronal loop}

We describe the coronal plasma by the ideal magnetohydrodynamic equations:

$\frac{\partial \varrho}{\partial t}+\nabla \cdot(\varrho \boldsymbol{V})=0$,

$\frac{\partial(\varrho \boldsymbol{V})}{\partial t}+\nabla \cdot[(\varrho \boldsymbol{V}) \boldsymbol{V}]=-\nabla p+\frac{1}{\mu}(\nabla \times \boldsymbol{B}) \times \boldsymbol{B}$,

$\frac{\partial p}{\partial t}+\nabla \cdot(p \boldsymbol{V})=-p(\gamma-1) \nabla \cdot \boldsymbol{V}$,

$\frac{\partial \boldsymbol{B}}{\partial t}=\nabla \times(\boldsymbol{V} \times \boldsymbol{B})$,

$\nabla \cdot \boldsymbol{B}=0$

where $\varrho$ is the mass density, $\boldsymbol{V}$ is the flow velocity, $\boldsymbol{B}$ is the magnetic field, $p$ is the pressure, $\mu$ is the magnetic permeability, and $\gamma=5 / 3$ is the adiabatic index. 
In this paper we limit our discussion to a two-dimensional magnetically structured atmosphere in which the plasma quantities are independent of the spatial coordinate $y, \partial / \partial y=0$, and $V_{y}=B_{y}=0$. This limitation, in combination with the chosen geometry (see below), removes the Alfvén wave from the system. Additionally, we assume that the initial, unperturbed (i.e. equilibrium) magnetic field is directed in the $z$-direction and approximates a coronal loop by smooth plasma profiles in which the inhomogeneity occurs in the $x$-direction only; i.e. $\boldsymbol{B}_{0}=\left(0,0, B_{0}(x)\right)$. In particular, we consider a loop in the form of a slab with a half-width $a$, field strength $B_{\mathrm{i}}$ at the center of the slab and mass density $\varrho_{0}$, embedded in a magnetic environment with field strength $B_{\mathrm{e}}$ and mass density $\varrho_{\mathrm{e}}$ reached at infinite distance from the slab axis. The equilibrium mass density profile $\varrho_{0}(x)$, is chosen as

$\varrho_{0}(x)=\varrho_{\mathrm{e}}+\left(\varrho_{\mathrm{i}}-\varrho_{\mathrm{e}}\right) \operatorname{sech}^{4}(x / a)$,

while for the magnetic field profile we adopt

$B_{0}(x)=B_{\mathrm{e}}+\left(B_{\mathrm{i}}-B_{\mathrm{e}}\right) \operatorname{sech}^{4}(x / a)$,

which corresponds to an open magnetic structure.

The equilibrium magnetic field, $B_{0}(x)$, and pressure $p_{0}(x)$ profiles must satisfy the total pressure balance condition

$\frac{\mathrm{d}}{\mathrm{d} x}\left(p_{0}(x)+\frac{B_{0}^{2}(x)}{2 \mu}\right)=0$,

from which $p_{0}(x)$ is obtained. Hence, in equilibrium the total (gas plus magnetic) pressure is constant.

Instead of specifying $B_{\mathrm{i}}$ and $B_{\mathrm{e}}$ or $\varrho_{\mathrm{i}}$ and $\varrho_{\mathrm{e}}$ separately, we divide Eq. (6) by $\varrho_{\mathrm{e}}$, Eqs. (7) and (8) by $B_{\mathrm{e}}$ and introduce the density ratio $d$ and the ratio of Alfvén speeds $v$

$d \equiv \frac{\varrho_{\mathrm{i}}}{\varrho_{\mathrm{e}}}, \quad v \equiv \frac{c_{\mathrm{Ae}}}{c_{\mathrm{Ai}}}$,

where $c_{\mathrm{Ae}}=B_{\mathrm{e}} / \sqrt{\mu \varrho_{\mathrm{e}}}$ and $c_{\mathrm{Ai}}=B_{\mathrm{i}} / \sqrt{\mu \varrho_{\mathrm{i}}}$. Henceforth, we choose and hold fixed

$d=3.89, \quad v=2$.

These values correspond to a typical EUV coronal loop (Aschwanden 2003). They imply that $B_{\mathrm{i}}=0.986 B_{\mathrm{e}}$.

Introducing normalized variables, we measured mass density in units of $\varrho_{\mathrm{e}}$, temperature in $T_{\mathrm{e}}$, spatial variables in $a$ and time in units of the Alfvén transit time $t_{\mathrm{A}}=a / c_{\mathrm{Ae}}$.

Although the model described above is not completely physically justifiable, because we neglect the effects of gravity, field line curvature and non-circular cross-sections, it is good enough to reveal the presence of characteristic mass density oscillations, and thus should show the scenario which should take place in a more realistic three-dimensional model. It is also well known that in the limit of long wavelength waves the properties of symmetric waves in magnetic slabs and tubes are quite similar, while the properties of kink waves are different. In particular, the phase-speed of the lowest order non-symmetric wave propagating along the cylinder is $c_{k}$, where

$c_{k}=\sqrt{\frac{\varrho_{\mathrm{i}} c_{\mathrm{Ai}}^{2}+\varrho_{\mathrm{e}} c_{\mathrm{Ae}}^{2}}{\varrho_{\mathrm{i}}+\varrho_{\mathrm{e}}}}, \quad c_{\mathrm{Ai}}<c_{k}<c_{\mathrm{Ae}}$.
In the slab this phase speed is $c_{\mathrm{Ae}}$. Since we consider nonsymmetric perturbations, our results are strictly valid for a slab geometry only. However, we expect that wave propagation in real three-dimensional structures would not differ significantly from the present scenario in spite of the fact that the nonsymmetric wave would propagate with $c_{k}<c_{\mathrm{Ae}}$.

\subsection{Linear equations}

Small amplitude perturbations of the equilibrium described above obey the following equations:

$\varrho_{0}\left(\frac{\partial^{2}}{\partial t^{2}}-c_{\mathrm{A}}^{2} \frac{\partial^{2}}{\partial z^{2}}\right) V_{x}=-\frac{\partial}{\partial x}\left(\varrho_{0} c_{\mathrm{A}}^{2} \frac{\partial V_{z}}{\partial z}-\varrho_{0} c_{f}^{2} \nabla \cdot \boldsymbol{V}\right)$,
$\frac{\partial^{2} V_{z}}{\partial t^{2}}=c_{\mathrm{s}}^{2} \frac{\partial}{\partial z}(\nabla \cdot \boldsymbol{V})$,
$\frac{\partial \delta \varrho}{\partial t}=-\nabla \cdot\left(\varrho_{0} \boldsymbol{V}\right)$,

where the perturbed mass density $\delta \varrho=\varrho-\varrho_{0}, c_{\mathrm{s}}(x)=$ $\sqrt{\gamma p_{0} / \varrho_{0}}$ and $c_{f}(x)=\sqrt{c_{\mathrm{s}}^{2}+c_{\mathrm{A}}^{2}}$ are the sound and fast speeds, respectively.

Equations (12) and (13) describe the fast and slow magnetosonic waves, which are coupled. The fast modes of plasma structures can be strongly dispersive when their wavelength is comparable with the width of the structure, i.e. $2 a$ in the present case, while the slow waves are only weakly dispersive. In the low $\beta$ limit the slow wave propagates with a speed that is close to the sound speed in the loop.

From Eq. (14) it follows that in the linear limit the mass density $\delta \varrho$ evolves in time as a consequence of the non-zero velocity components $V_{x}$ and $V_{z}$. On the other hand, $\delta \varrho$ does not influence plasma evolution as $\delta \varrho$ does not enter the equations for $\boldsymbol{V}, \boldsymbol{\delta} \boldsymbol{B}$ and $p$. In this sense Eq. (14) decouples from the other equations.

\subsubsection{The limit of cold plasma}

It is instructive to envisage first the limit of cold plasma for which the gas pressure terms are neglected. In equilibrium the magnetic field is constant, $B_{0}=B_{\mathrm{e}}$. Small amplitude oscillations are governed by the linear Eqs. (12)-(14) with the gas pressure terms dropped. These equations then simplify to

$$
\begin{aligned}
& \varrho_{0}\left(\frac{\partial^{2}}{\partial t^{2}}-c_{\mathrm{A}}^{2} \frac{\partial^{2}}{\partial z^{2}}\right) V_{x}=\frac{\partial}{\partial x}\left(\varrho_{0} c_{\mathrm{A}} \frac{\partial V_{x}}{\partial x}\right), \\
& \frac{\partial \delta \varrho}{\partial t}=-\frac{\partial\left(\varrho_{0} V_{x}\right)}{\partial x}
\end{aligned}
$$

As a consequence of the cold plasma assumption the slow wave is removed from the system and Eqs. (15) and (16) describe respectively the fast waves and the mass density perturbations associated with them.

\section{Impulsively generated MHD waves}

Consider a coronal loop described by Eqs. (6) and (7). Perturbations can be excited in such a loop in numerous ways. 
Here we focus our attention on impulsively excited waves. We launch a pulse in the $x$-component of the velocity having the form

$V_{x}(x, z, t=0)=\frac{V_{x 0}}{\cosh ^{2}[(x+5 z) / a] \cosh ^{2}(z / a)}$,

where $V_{x 0}$ is the amplitude of the pulse. The component of velocity parallel to the field, $V_{z}(t=0) \equiv 0$, and $\boldsymbol{B}$ is not perturbed. This pulse expands rapidly and upon hitting the loop excites a packet of waves in which fast kink waves have the highest contribution.

We now consider three cases separately: (a) small amplitude cold plasma oscillations, (b) small amplitude warm plasma oscillations and (c) large amplitude warm plasma oscillations. In case (a) all gas pressure terms can be neglected compared to magnetic terms because the plasma $\beta=0$. For cases (b) and (c) these terms have to be retained in the MHD equations although they are not as important as the magnetic field terms because $\beta \ll 1$. For case (a) the oscillations are described by the linear Eqs. (15)-(16). In both cases the plasma equations are solved numerically in an Eulerian box with the $x$ and $z$ dimension $(-30,20)$ and $(-25,25)$ (or $(-50,50)$ in case $(c))$, respectively, in units of $a$. Open boundary conditions were used in the case of warm plasma and periodic ones in the case of cold plasma because of FFT code employed (Murawski \& Roberts 1993). This implies that in both cases we consider the central portion of an infinitely long loop.

For cases (b) and (c) MHD Eqs. (1)-(5) are solved numerically using the FLASH code (Zingale et al. 2002) which is a nice tool for numerically solving MHD equations. The high-order Riemann solver of Roe type that is implemented in this code yields accurate results near steep gradients and moving contact discontinuities. The MHD part uses Powell's method (Powell 1999) which allows the magnetic field to be kept divergence-free to machine-dependent round-off errors. The computer code is formally second-order accurate in space and time.

\subsection{Small amplitude cold plasma oscillations}

In this section we discuss linear perturbations of cold plasma which are described by Eqs. (15) and (16). These linear equations allowed us to eliminate numerical errors which are associated with an inhomogenous equilibrium. In the nonlinear case we usually have to work with the total field $\varrho$ which contains the inhomogeneous background $\varrho_{0}(x)$ and perturbations $\delta \varrho(x, z, t), \varrho=\varrho_{0}+\delta \varrho$. Because of various numerical effects there are often some small departures of a numerical equilibrium from the analytical one, $\varrho_{0}$. Due to these departures a spurious signal propagates when one starts from the analytical equilibrium until the equilibrium approaches its numerical limit. As a consequence of its simplicity and of the fact that there are numerical errors that are associated with the representation of the inhomogeneous equilibrium, the case of cold plasma will serves as a reference for more complex models of loop oscillations. This role as a reference is strengthened by the fact that independent codes are employed for the cold and warm plasma computations.
Figure 1 displays spatial profiles of the perturbed mass density $\delta \varrho$ of cold plasma oscillations at $t=25$. These profiles have been obtained with the use of the spectral fast Fourier code (Murawski \& Roberts 1993). The initial pulse was launched at $(-5 a, 0)$, while Murawski \& Roberts (1993) generated the pulse at $(0,0)$.

As a result of the initial impact in the region $x<0(x>0)$ $\delta \varrho<0(\delta \varrho>0)$ in the sense that the loop is shifted as a whole and two ridges in $\delta \varrho(x)$ at the sides of the loop appear, i.e. the loop is pushed to greater $x$-values (away from the pulse). Note that along the loop this displacement is almost independent of $z$, once it is set up by the above wave trains.

To explain the behaviour of $\delta \varrho$ at the slab edges we consider the term $-\varrho_{0, x} V_{x}$ which is dominant there. At the left (right) edge $\varrho_{0}$ grows (decreases) with $x$ and $\varrho_{0, x}$ is positive (negative) there. As $V_{x}$ is positive the r.h.s. of Eq. (16) is negative (positive) and $\delta \varrho$ declines (grows) with time at the left (right) edge. As a consequence of this at the early stage of the evolution of the waves we have a valley (peak) in $\delta \varrho$ at the left (right) edge. This results in the slab moving from the negative $x$ direction (bottom panel of Fig. 1).

\subsection{Warm plasma oscillations}

We now consider a warm plasma for which the pressure terms have to be retained in the MHD Eqs. (1)-(5). We specify the plasma $\beta$ as $\beta \equiv 2 \mu p_{\mathrm{e}} / B_{\mathrm{e}}^{2}$. The mass density contrast $d$ and the Alfvén speed ratio $v$ are defined by Eq. (10). For this choice we have the plasma $\beta=0.003$.

For all numerical runs we apply free-boundary conditions at the boundaries of the simulation region. This choice of boundary conditions reflects the fact that the real medium extends well beyond the computational domain. Thus we assume that MHD waves have no time to travel the entire length of the loop and that they have not reached the ends of the loop, where the dense lower atmosphere causes reflection, and so the wave propagates freely as if the structure were open. Since we consider only open structures, i.e. we neglect the reflection from the boundaries, our solution is valid only in a finite time interval. Assuming a typical loop length $L=10^{5} \mathrm{~km}$ and the Alfvén speed $c_{\mathrm{Ae}}=10^{3} \mathrm{~km} \mathrm{~s}^{-1}$, we can estimate the time $t_{v}$ after which our solution cannot be applied to a real bounded structure as $t_{v}<\frac{1}{2} L / c_{\mathrm{Ae}}=50 \mathrm{~s}$.

\subsubsection{Small amplitude waves}

Figure 2 displays the spatial profiles of the perturbed mass density $\delta \varrho$ at $t=25$. These profiles resemble the result of Fig. 1 . As a consequence of the finite value of the plasma $\beta$ the slow wave is present in the system. Such a wave is seen in Fig. 2 in the neighborhood of the point $x=-5, z=0$ in the form of two local pulses which resulted from the splitting of the original pulse. In general, the results are very similar to those of the cold plasma case: oppositely directed disturbances propagate along the loop (top panel of Fig. 2). In this case the perturbed mass density profile $\delta \varrho$ reveals the presence of two ridges. Such a distribution of density results in the conclusion that plasma in 

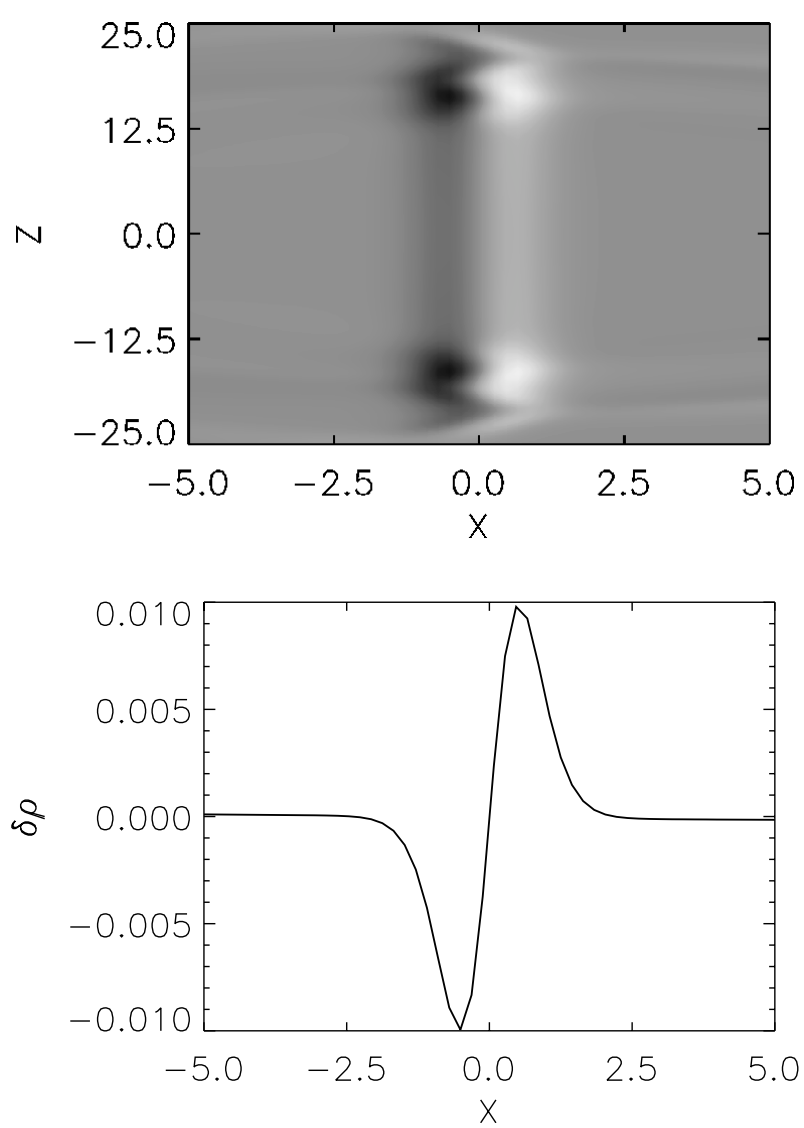

Fig. 1. The spatial profile of the perturbed mass density $\delta \varrho(x, z, t=25)$ (top panel) and its cross-section at $z=5$ (bottom panel). These profiles correspond to the limit of cold plasma.

the loop is spread asymmetrically. This behaviour can be explained in a similar way as in the case of the cold plasma. At the initial stage the term $-\varrho_{0, x} V_{x}$ is dominant in Eq. (14). At later times the other two terms of the r.h.s. of Eq. (14), as well as the nonlinear terms in Eq. (1) contribute to the scenario of $\delta \varrho$ evolution in time and make $\delta \varrho$ more complex. This contribution results in loop movement as a whole.

The initial pulse of Eq. (17) produces complex time signatures which are obtained collecting in time wave signals at the detection point (Roberts et al. 1984; Murawski \& Roberts 1993; Nakariakov et al. 2004). Figure 3 shows time signatures which are obtained collecting the perturbed mass density at different spatial points. It is discernible that with a larger distance from the exciter time signatures are shorter and more complex. These time signatures reveal time scales of the order of a dozen or so seconds.

Note that Fig. 3 shows a rapidly decaying oscillation with a very short period at the center of the slab. This oscillation results from a natural decaying response of the medium to an impulsive perturbation. The counterparts of these rapid oscillations are seen in Figs. 1 and 2 between $|z|=15$ and $|z|=25$. One can see the attenuated oscillation at the boundary between black and white signal which corresponds to a fast magnetosonic wave, propagating with the Alfvén speed. The signal gets more complex as it moves out as a consequence of dispersion which spreads the wave packet.
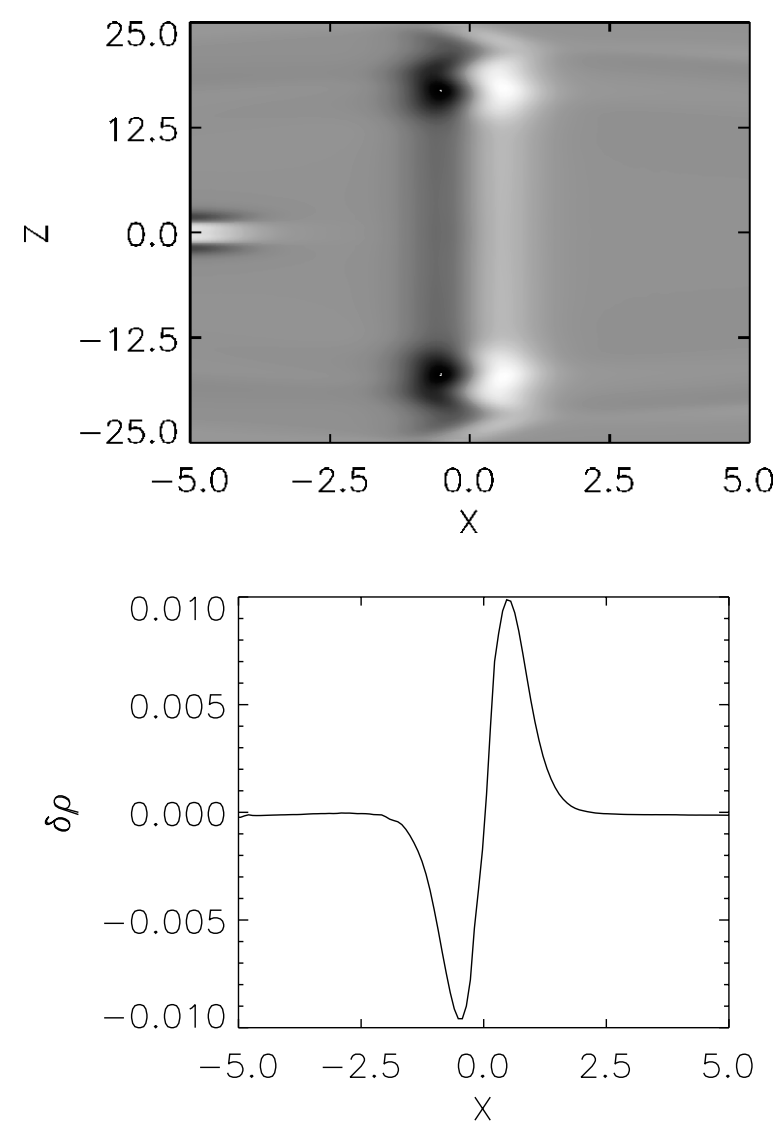

Fig. 2. The spatial profile of the perturbed mass density $\delta \varrho(x, z, t=25)$ for the initial amplitude $V_{x 0}=0.1$ (top panel) and its cross-section at $z=5$ (bottom panel) in the case of warm plasma. The position of the initial pulse $(-5,0)$ is visible.

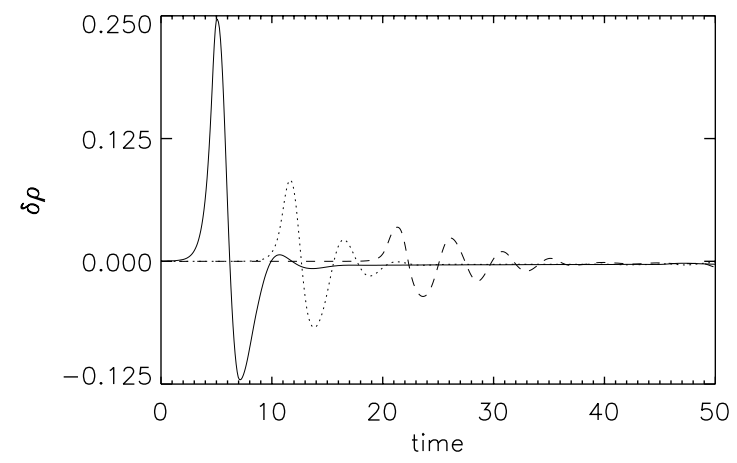

Fig. 3. Time signatures which are obtained by measuring the mass density at the detection point $x=0.0, z=0$ (solid line), $z=10$ (dotted line), $z=20$ (dashed line), for the initial amplitude $V_{x 0}=0.1$.

\subsubsection{Large amplitude waves}

As the loop is slightly shifted in the case of $V_{x 0}=0.1$ we cannot observe this shift directly but we can only measure the perturbed mass density $\delta \varrho$. Note that this shift increases with the amplitude of the pulse (Fig. 4) We can observe that for superAlfenic pulse amplitudes $V_{x 0}=2.0$ and $V_{x 0}=5.0$ the scenario does not change very much. 

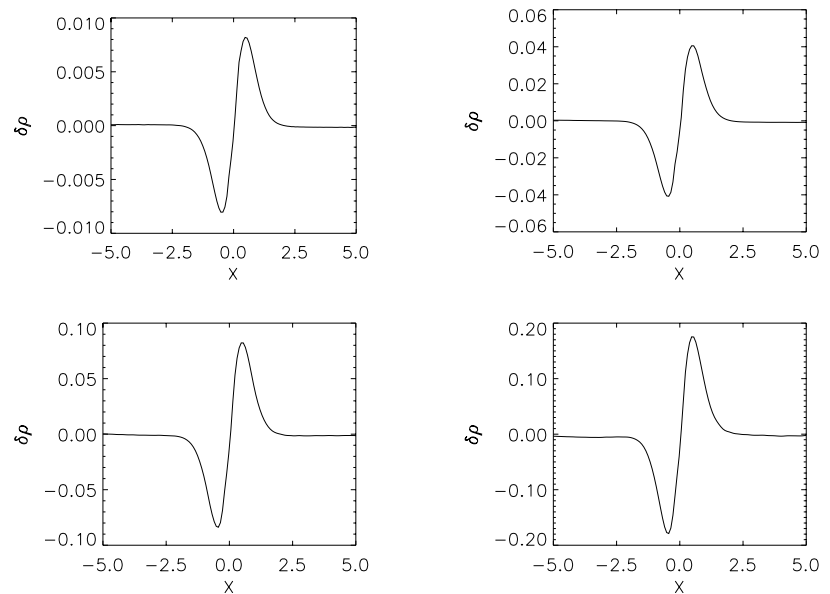

Fig. 4. The cross-section of the perturbed mass density $\delta \varrho(x, z=$ $12.5, t=30$ ) in the case of $V_{x 0}=0.1$ (left top panel), $V_{x 0}=0.5$ (right top panel), $V_{x 0}=1.0$ (left bottom panel) and $V_{x 0}=2.0$ (right bottom panel).

One way to cause a perturbation that would make a significant shift of the whole loop is to launch a pulse in density, pressure and velocity, e.g.

$$
\begin{gathered}
V_{x}(x, z, t=0)=\frac{V_{x 0}}{\cosh ^{2}[(x+10) / a] \cosh ^{2}(z / a)}, \\
\varrho(x, z, t=0)=\frac{\varrho_{x 0}}{\cosh ^{2}[(x+10) / a] \cosh ^{2}(z / a)}, \\
p(x, z, t=0)=\frac{p_{x 0}}{\cosh ^{2}[(x+10) / a] \cosh ^{2}(z / a)} .
\end{gathered}
$$

Here $\varrho_{x 0}$ and $p_{x 0}$ denote amplitudes of the pulse in mass density and pressure, respectively. We choose the following values of these amplitudes: $V_{x 0}=2.0, \varrho_{x 0}=2.0, p_{x 0}=2.0$.

Figure 5 shows the evolution of a kink wave propagating along the loop. At the initial stage of the simulation the loop is hit by the approaching signal and strongly shifted to the right. After a few seconds the pulse causes two perturbations that propagate upwards and downwards along the loop in the form of a kink wave.

The shift of the loop as a whole is shown in Fig. 6 which displays mass density $\varrho(x, z=5, t)$ at given moments of time. We observe that the loop is initially shifted very strongly to the right (dashed line) and after some time it moves back towards its initial position (dotted line).

As the chosen values of the pulse amplitude are large the time signatures are more complex than in the weak amplitude case (Fig. 3).

\section{Summary}

We have developed a simple two dimensional model of the coronal plasma that makes no allowance for loop curvature, stratification, nor is there any realistic modeling of the coupling of the coronal plasma to the denser layers of the solar atmosphere. Using this model we have numerically simulated the response of the coronal plasma to the impulsively generated MHD waves.
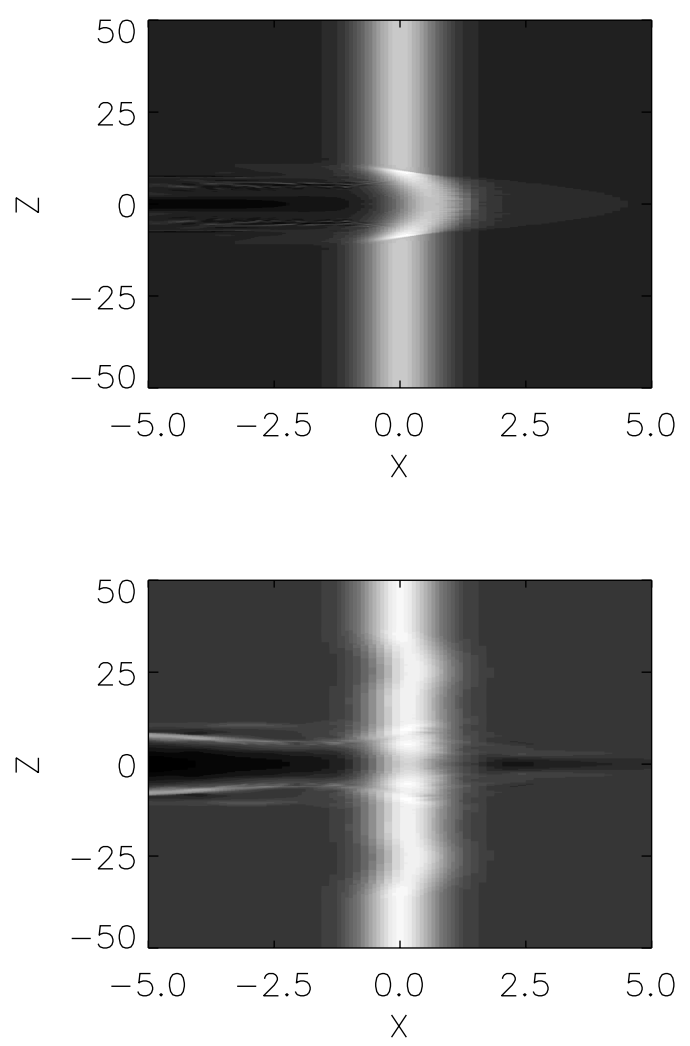

Fig. 5. The mass density $\varrho(x, z, t=10)$ (top panel) and $\varrho(x, z, t=35)$ (bottom panel) in the case of $V_{x 0}=2.0, \varrho_{x 0}=2.0$ and $p_{x 0}=2.0$.

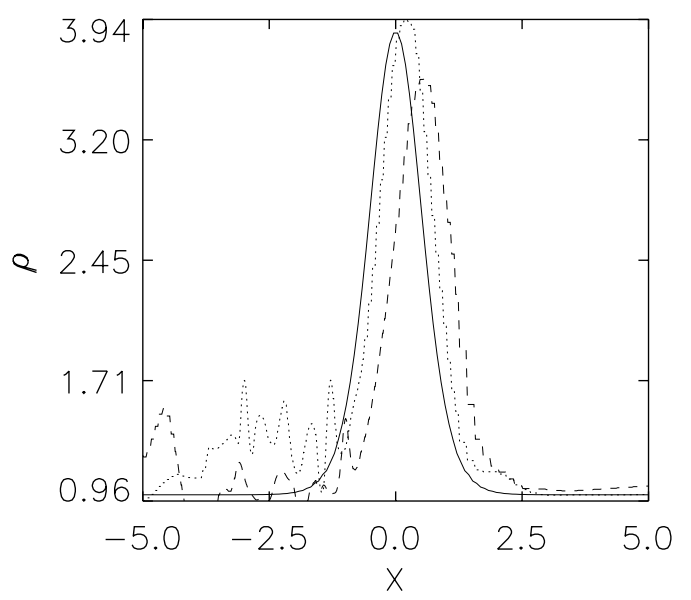

Fig. 6. The cross-section of the mass density $\varrho(x, z=5.0, t=0)$ (solid line), $\varrho(x, z=5.0, t=15)($ dashed line $)$ and $\varrho(x, z=5.0, t=25)$ (dotted line) in the case of $V_{x 0}=2.0, \varrho_{x 0}=2.0$ and $p_{x 0}=2.0$.

Our main and new results are:

1) An initial pulse excites perturbations in the mass density all over the loop. At the initial stage of their time evolution the amplitude of these perturbations can be essentially constant along the loop. This is interesting as it appears that a mass density contains the finger-prints of the initial perturbation.

2) As a consequence of the asymmetric mass density profiles the plasma in the loop is heated asymmetrically. At the initial stage of pulse development it is heated (cooled) on the down-wind (up-wind) side. 


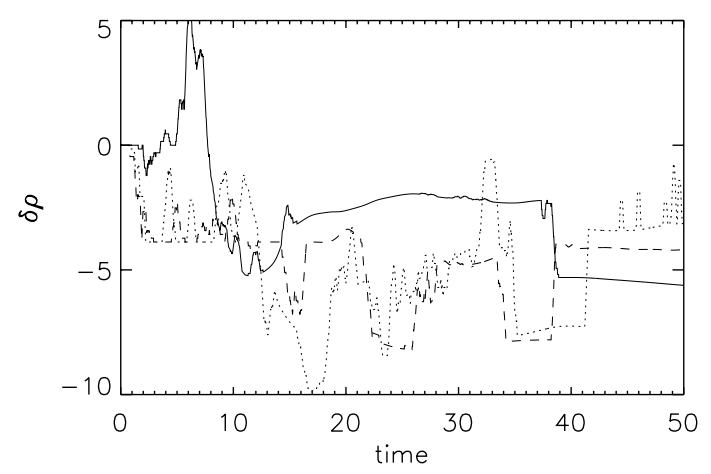

Fig. 7. Time signatures which are obtained by measuring the mass density at the detection point $x=0.0, z=0$ (solid line), $z=10$ (dotted line), $z=20$ (dashed line), for $V_{x 0}=2.0, \varrho_{x 0}=2.0$ and $p_{x 0}=2.0$.

As both mass density and temperature fluctuations can be detected observationally a sudden line intensity brightening at one side of a coronal loop can be a proof that an impulsive energy release took place a while ago in the ambient medium on this side of the loop (Wang \& Solanki 2004).

This finding is important from the point of view of future solar missions (Solar-B, STEREO, SDO and Solar Orbiter) whose instrumental capabilities would make it possible to detect possible asymmetries in the wave profiles.

Acknowledgements. The authors express their cordial thanks to Prof. Sami K. Solanki and Dr. Valery Nakariakov for their helpful comments on an earlier version of this paper and Dr. Mike Ruderman for his constructive comment. This work was financially supported by the grant from the State Committee for Scientific Research Republic of Poland, with KBN grant No. 2 PO3D 016 25. The software used in this work was in part developed by the DOE-supported ASCI/Alliance Center for Astrophysical Thermonuclear Flashes at the University of Chicago.

\section{References}

Aschwanden, M. 2003, in Proc. NATO Workshop, Turbulence, waves and instabilities in the solar plasma, Nato Sc. Ser., II. Math. Phys. Chem., 124, ed. R. Erdélyi, K. Petrovay, B. Roberts, \& M. Aschwanden

Cooper, F. C., Nakariakov, V. M., \& Williams, D. R. 2003, A\&A, 409, 325

Murawski, K., Aschwanden, M., \& Smith, J. M. 1998, Sol. Phys., 179, 313

Murawski, K., \& Roberts, B. 1993, Sol. Phys., 144, 255

Murawski, K., \& Roberts, B. 1994, Sol. Phys., 151, 305

Nakariakov, V. M. 2003, in The Dynamic Sun, ed. B. Dwivedi (CUP)

Nakariakov, V. M., \& Roberts, B. 1995, Sol. Phys., 159, 399

Nakariakov, V. M., Arber, T. D., Ault, C. E., et al. 2004, MNRAS, 349, 705

Powell, K. 1999, J. Comp. Phys., 154, 284

Roberts, B. 2000, Sol. Phys., 193, 139

Roberts, B., Edwin, P. M., \& Benz, A. O. 1984, ApJ, 279, 857

Wang, T. J. 2004, in Proc. of SOHO13 Waves, Oscillations and SmallScale Transient Events in the Solar Atmosphere: A Joint View from SOHO and TRACE, ESA SP-547, January 2004

Wang, T. J., \& Solanki, S. K. 2004, A\&A, submitted

Zingale, M., Dursi, L. J., Zuttane, J., et al. 2002, ApJS, 143, 539 